\section{gynécologie suisse Société Suisse de Gynécologie et d'Obstétrique Schweizerische Gesellschaft für Gynäkologie und Geburtshilfe Società Svizzera di Ginecologia e Ostetricia}

http://www.sggg.ch

Präsident

Prof. Dr. méd. David Stucki

Clinique de gynécologie et obstétrique

Hôpital cantonal Fribourg

Route de Bertigny

CH-1708 Fribourg (Suisse)

Tel. +4126426 73 65, Fax +41264267367

E-Mail stuckid@hopcantfr.ch
Sekretär

Prof. Dr. med. Mario Litschgi

Kantonsspital Schaffhausen

CH-8208 Schaffhausen (Schweiz)

Tel. +41 5263423 15, Fax +41526342398

E-Mail mario.litschgi@kssh.ch

\section{Bericht des Präsidenten der Schweizerischen Gesellschaft für Gynäkologie und Geburtshilfe}

Bei der Übernahme eines Mandates ist die Standortbestimmung von Problemen vorrangig, welche aktuell bestehen und kurz- oder mittelfristig gelöst werden müssen. Nicht nur die Mannigfaltigkeit der Themen, sondern auch die Anzahl der Herausforderungen, welche mich tagtäglich erreichen, ist beeindruckend. Per Telefon, per Post, aber vor allem per E-Mail soll man jederzeit und immer zur Verfügung stehen. Oft müssen «Nacht- und Nebelaktionen» gestartet werden, um möglichst schnell zu handeln bzw. den korrekten Ablauf von Prozessen zu gewährleisten. Diese Art der Arbeit ist für mich neu und hat mich gelehrt, die Abläufe meiner täglichen Arbeit zu hinterfragen und teilweise zu ändern.

Die Vorstandssitzungen, welche alle 6-8 Wochen stattfinden, beinhalten eine beeindruckende Traktandenliste, deren einzelne Punkte in konzentrierter Arbeit über mehrere Stunden bearbeitet werden. Hier möchte ich die engagierte und dynamische Mitarbeit meiner Vorstandskollegen speziell hervorheben, welche bereit sind, anstehende Probleme anzupacken, zu diskutieren und nach einer optimalen Lösung zu suchen. Hervorheben möchte ich speziell die Arbeit unseres Generalsekretärs, dem es gelingt, dank perfektester Organisation, stets den Überblick zu bewahren.

Die Vielsprachigkeit unseres Landes mit seinen Feinheiten, aber auch spezifischen Interessen bringt eine spezielle Farbe in alle zu behandelnden Themen. Gerne möchte ich Ihnen im Folgenden eine Auswahl der aktuellen Themen vorstellen.

\section{Managed Care}

Dieses Thema wurde anlässlich der Sitzung der Ärztekammer im Dezember 2006 ausgiebig diskutiert. Managed care wurde als Alternative zur Aufhebung des Kontrahierungszwangs vorgestellt. Die Bildung von «Ärzte-Netzwerken» scheint das Allheilmittel in den Augen der Politiker zu sein. Die FMH hat eine Zusammenstellung dieser Netzwerke erarbeitet und möchte die kantonalen Gesellschaften beauftragen, die Realisation derselben durchzuführen.

Ohne bezüglich Rechtmässigkeit/Erfolg solcher Beispiele im Ausland ins Detail zu gehen, ist es für uns Frauenärzte äusserst wichtig, den Politikern klar aufzuzeigen, dass wir schon seit längerer Zeit eine gewisse Hausarztrolle für die Frauen einnehmen. Dies nicht nur auf dem Gebiet der Prävention, sondern für alle
Krankheiten der Frau. Eine Motion, welche ich in diesem Sinne ausgearbeitet hatte, wurde intensiv debattiert, aber schlussendlich vom Plenum der Ärztekammer verworfen. Die heftige Diskussion hat nicht nur unserer Fachgesellschaft die Komplexität des Sujets aufgezeigt, sondern ebenfalls beispielsweise den Psychiatern und den Ophthalmologen, welche alle den Status des Hausarztmediziners fordern. Ganz klar müssen wir uns in diesem Sinne in den Diskussionen auf Niveau des Bundes, der Kantone und der Regionen engagieren und positionieren. Den Zürcher Gynäkologen möchte ich für deren tatkräftige Unterstützung bezüglich dieses strategisch wichtigen Themas danken.

\section{Weiterbildungsprogramm}

Dem neuen Weiterbildungsprogramm wurde anlässlich der letzten Schweizerischen Chefärztekonferenz im Dezember 2006 der letzte Schliff verpasst und somit wird es nun den Hauptanliegen - EU-Kompatibilität und Anpassung an die neuen Gegebenheiten in unserem Fach - gerecht. Zusammengefasst sieht es folgendermassen aus: Die Dauer zur Ausbildung des BasisGynäkologen (Sockel-Gynäkologen) beträgt 5 Jahre. Nach Ablauf dieser Zeit sollte ein junger Facharzt fähig sein, in einer Praxis die gynäkologische Grundversorgung zu garantieren. Damit dies gewährleistet ist, beinhaltet die Grundausbildung die Möglichkeit von maximal $2 \times 6$ Monaten Weiterbildung in einer gynäkologischen Praxis.

Wer anschliessend als operativ tätiger Gynäkologe in einer Privatklinik oder an einem öffentlichen Spital tätig sein will, muss sich den Schwerpunktstitel «Interventioneller Gynäkologe» erarbeiten. Im Anschluss daran kann noch zusätzlich der Schwerpunktstitel «gynäkologische Onkologie» oder «Urogynäkologie» erlangt werden.

Voraussetzung für die Schwerpunktstitel «fetomaternelle Medizin» oder «Endokrinologie» und «Reproduktionsmedizin» ist die Basisausbildung. Mit grosser Ausdauer und viel Diplomatie hat Prof. Dr. Patrick Hohlfeld dieses neue Weiterbildungsprogramm ans Ziel gebracht. Ein herzliches Dankeschön dafür!

\section{Einteilung der Weiterbildungsstätten}

Die aktuelle Klassifikation in Kliniken A, B, C und D entspricht nicht mehr der Realität. Trotz Anpassungen, welche anlässlich von Visitationen oder bei Chefarztwechsel vorgenommen werden, muss die Klassifikation überarbeitet werden. Auf der Suche nach einem aktuellen und modernen Konzept hat sich, im 
Rahmen der Diskussion über das neue Weiterbildungsprogramm, die Idee der Bildung von Netzwerken entwickelt. Diese Netzwerke sollen sich aus mehreren Regional- und Kantonsspitälern und einer Universität bzw. einem grossen Kantonsspital (A-Klinik) zusammensetzen. Dieser neuen Einteilung werden viele Vorteile zugeschrieben: organisierte Rotation von Assistenten und Oberärzten, gemeinsame interne Weiterbildungen, optimale Ausnützung einzelner Ressourcen und, last but not least, Überwachung der Qualität der Auszubildenden. Die Schweizerische Chefärztekonferenz, unter der Präsidentschaft von Prof. Roland Zimmermann, hat nun die Aufgabe, dieses Projekt innerhalb der nächsten 2 Jahre umzusetzen und innerhalb einer Übergangsfrist zum Laufen zu bringen.

\section{ASF-Statistik}

Die Situation dieser, seit Jahren funktionierenden, gelegentlich aber auch hinterfragten Statistik wurde anlässlich der letzten Schweizerischen Chefärztekonferenz im Dezember 2006 in Bern erneut diskutiert.

Grundlage der erneuten Diskussion sind die durch die FMHStatuten und das KVG geforderten qualitätssichernden Massnahmen. Eine weitere Notwendigkeit, dieses Thema wieder aufzunehmen und zu aktualisieren, liegt im Problem der Berufspolitik. Es ist zunehmend wichtig, unsere Tätigkeit korrekt, glaubwürdig und flächendeckend zu dokumentieren.

Somit hat der Vorstand der SGGG mit Unterstützung der Schweizerischen Gynäkologischen Chefärztekonferenz beschlossen, die ASF-Statistik zu reaktualisieren. Dieses Arbeitswerkzeug wird modernisiert und soll leistungsfähig sein und sämtliche Weiterbildungsstätten in sich vereinigen. Dieses Ziel soll bis Anfang 2008 erreicht werden. Dr. Thomas Hess als Präsident der Arbeitsgruppe und Dr. Willi Marti werden «Dampf geben», damit dieses ehrgeizige Ziel erreicht werden kann.

\section{Diagnosis-Related Groups}

Es ist unvermeidlich, dass das neue pauschalierte Abrechnungssystem Swiss DRG eingeführt wird. Der Zeitpunkt ist nach letzten Meldungen 2010! Auf dem deutschen System beruhend, sind die Konsequenzen für die Spitalplanung und -finanzierung in unserem Land zweifellos von grosser Tragweite. Die Erfahrungen, welche in Nachbarländern gemacht wurden, geben ein gutes Beispiel dafür. Auf jeden Fall ist es äusserst wichtig, sogar zwingend, dass wir, d.h. alle im akut-somatischen Bereich tätigen Ärzte und Ärztinnen, in diesem Prozess mitsprechen können und miteinbezogen werden.

Der Verein Swiss DRG, in welchem Mitglieder von $\mathrm{H}+$, der Gesundheitsdirektorenkonferenz, der Santésuisse, der Zentralstelle für Medizinaltarife MTK und der FMH sitzen, ist im Moment daran, weitere Schritte auszuarbeiten (z.B. «case mix office»). Da die DRG vor allem den akut somatischen und stationären Bereich betreffen, ist es unabdingbar, dass wir mit der Person, welche uns als «FMH-Sitz» in diesem Verein vertritt, eng zusammenarbeiten. Noch besser wäre es, wenn die betreffende Person ein Vertreter der FMCH wäre. Aus diesem Grunde hat die FMCH eine eigene Arbeitsgruppe DRG gegründet.

Am letzten Jahreskongress unserer Fachgesellschaft in Interlaken wurde deshalb anlässlich der Mitgliederversammlung beschlossen, neu ein DRG-Departement im Vorstand einzuführen. Die damit beauftragte Person ist Dr. Dieter Musfeld, der sich intensiv und aufmerksam dem Thema DRG widmet.

\section{Qualitätskontrolle}

Dieses Thema wurde vor Jahren durch Prof. Urs Haller ins Leben gerufen. Aktuell wird die Qualitätssicherungskommission durch Prof. Daniel Surbek präsidiert. Die von uns in diesem Ge- biet gewählte Vorreiterrolle wird seit Jahren von der FMH positiv hervorgehoben. Jede Weisung, jeder Expertenbrief, jede Studie, jedes Referenzpapier wird sorgfältig durch diese Expertengruppe gefiltert. Dies erlaubt uns eine wirksame und präzise Qualitätskontrolle.

Das aktuell vordringlichste Thema, auf welches wir ungeduldig warten, ist die Stellungnahme zum Problem der Impfung gegen das Zervixkarzinom.

\section{Critical Incident Reporting System CIRS/CIRNET}

Dieses, seit vielen Jahren durch die Luftfahrt bekannte System wurde vor einigen Jahren in der Medizin durch die Anästhesisten eingeführt. Anonymisiert können Problemfälle oder solche Fälle, welche für den Patienten fast zu einem Problem wurden, gemeldet werden. Seit einigen Jahren steht es auch den Mitgliedern der SGGG zur Verfügung. Die Tatsache, dass auch unsere Nachbarländer, vor allem Deutschland und Österreich, mitmachen, erlaubt es uns, eine Sammlung von möglichen Gefahren für die Patienten anzulegen und diese anschliessend im Alltag und in der Ausbildung konstruktiv einzusetzen. Um zu vermeiden, dass jedes einzelne Spital ein eigenes System einführt, analysiert der Vorstand, ob sich die SGGG dem neuen System CIRNET, welches von den Patientenorganisationen ins Leben gerufen wurde, anschliessen soll.

\section{Tarife}

Dr. Pierre Villars, verantwortlich für das Departement Tarife, hat sich in letzter Zeit als harter Fighter in Szene gesetzt und musste oft sehr schnelle Entscheide treffen. Dies war vor allem im Fall der Reorganisation des Kapitels 30 vom Tarmed-Vertrag wichtig, denn hier ging es im Rahmen des Reengineering III um die Abgeltung des gynäkologischen Ultraschalls. In den zähen Verhandlungen, in welchen die SGGG durch die SGUM vertreten wurde, konnte der FMH klar signalisiert werden, dass wir mit der Anwendung des Modells «Korig» anstelle des Models «Infra» zur Leistungsabrechnung nicht einverstanden sind. Schlussendlich wurde das Problem erkannt, und aktuell befinden wir uns an einem toten Punkt.

Bezüglich eines weiteren Verrechnungsproblems des Tarmed, nämlich der Abrechung für die therapeutische Hysteroskopie, kann die Assistenz nicht mehr ohne weiteres abgerechnet werden. Der nachweisbare Bedarf für eine Assistenz kann aber immer noch abgerechnet werden.

\section{Foederatio Medicorum Chirurgicorum Helvetica}

Die Integration unserer Gesellschaft unter diesem neuen Dachverband ist gut angelaufen. Prof. Mario Litschgi wurde in den Vorstand gewählt und wird zusätzlich FMCH-Delegierter in der FMH sein. Ich gratuliere ihm zur Wahl und danke ihm bereits jetzt für die zusätzliche Arbeit, die er für uns leisten wird.

Die in den ersten gemeinsamen Sitzungen besprochenen Themen liessen mich eine grosse Übereinstimmung gemeinsamer Interessen, aber auch Sorgen, erfahren. Gemäss dem Sprichwort «Gemeinsam sind wir stark» stellt meiner Meinung nach bereits heute unser Beitritt zur FMCH einen Gewinn dar. Themen wie etwa Haftpflichtversicherung, Ausbildung unseres Nachwuchses, Einführung der DRG, Tarmed oder Managed care zeigen eine Auswahl unserer aktuellen «Kriegsschauplätze».

\section{Kontinuierliche Fortbildung}

Die neue Fortbildungsordnung ist revidiert und von der KWFB einstimmig genehmigt. Die Akkreditierung dieses meiner Meinung nach exzellenten Programms ist auf 7 Jahre festgelegt. Auch hier hat die SGGG eine Pionierrolle eingenommen. 
Der genaue Text kann unter www.sggg.ch (unter «Akademie», «kontinuierliche Fortbildung») eingesehen werden.

\section{Selbstdeklaration}

Zwei Möglichkeiten der Selbstdeklarationen existieren: Die erste Möglichkeit betrifft den Gynäkologen als Einzelperson, die andere Möglichkeit betrifft die Klinik als Ausbildungsstandort. Unter «my fmh» konnte jeder Kollege bis zum 1. Januar 2007 seine eigenen Leistungen, welche er regelmässig erbringt und durch Fortbildungen auszuweisen hat, der FMH mitteilen. Auf der Basis dieser Eingabe wird die personenbezogene, zukünftige Besitzstandsgarantie festgelegt. Ich bedanke mich bei Dr. Christoph Winkler für das Publizieren einer Zusammenfassung des korrekten Vorgehens auf der Homepage der SGGG. Die Selbstdeklaration geschieht auf völlig freiwilliger Basis.

In Analogie zu dieser Deklaration der Einzelperson existiert eine Deklaration der Weiterbildungsstätten (Klinikprofil GEM. ASF-Daten).

\section{Virtuelles Training}

Die Gesellschaft für Gynäkologie und Geburtshilfe ist im Besitz eines Sonotrainers und eines Lapsim (Trainer für die Laparoskopie). Da der Sonotrainer relativ stabil ist, kann er problemlos transportiert und somit vermietet werden. Ein Reglement für die Versicherungsbedingungen des Transports und des Aufenthalts am Übungsort ist in Erarbeitung. Gleichzeitig werden die Preise für die Miete festgelegt. Die Beschlüsse werden auf der Homepage der SGGG publiziert werden.

Trotz Einspruchs der Mitglieder der AGE, welche auch den Lapsim zur Miete freigeben wollte, hat der Vorstand beschlossen, diesen definitv im Swiss-Endos-Zentrum in Fribourg zu stationieren, um Transportschäden an den sehr fragilen Simulatoren $\mathrm{zu}$ vermeiden. Selbstverständlich wird es für alle Interessierten die Möglichkeit geben, sich an offiziellen Fortbildungstagen am virtuellen Modell und am Pelvitrainer zu schulen.

\section{Deutsch-Amerikanisches Forum}

Anlässlich des letzten Jahreskongresses der DGGG wurde dieses Forum gegründet. Prof. Wolfgang Holzgreve wurde als Vizepräsident gewählt und ist in unserem Vorstand für das Departement internationale Zusammenarbeit zuständig.

FIGO 2012

Der nächste FIGO-Kongress wird also 2009 in Südafrika, derjenige von 2012 in Rom stattfinden.

Voller Enthusiasmus ist eine Delegation unseres Vorstandes nach Kuala Lumpur gereist, um den FIGO-Kongress von 2012 nach Genf zu holen. Leider sind wir, trotz professioneller Unterstützung des Tourismusbüros von Genf, der Stadt Rom unterlegen. Die touristische Attraktivität und die plötzlich niedrigeren Kosten für das Kongresszentrum in Rom waren der Grund unserer Niederlage.

\section{Franchise}

Die SGGG setzt sich für die Franchisebefreiung bei den Schwangerschaftskontrollen ein. Die aktuelle Situation sieht vor, dass nur eine problemlose Schwangerschaft von der Franchise befreit ist. Sobald Komplikationen auftreten, fallen diese Kosten unter die Franchise. Paradoxerweise wird also eine Frau mit einer Risikoschwangerschaft zusätzlich durch die anfallenden Kosten bestraft.

Mit Hilfe von politischen Gruppen konnte eine Motion lanciert werden, welche aktuell beim Bundesrat liegt. Ich hoffe, dass dieses politisch wichtige Thema möglichst bald von unserer
Regierung behandelt und akzeptiert wird. Sämtlichen Beteiligten danke ich für ihren Einsatz.

\section{Swissmedic}

Swissmedic hat eine Strafverfolgung gegen einen unserer Kollegen eröffnet, da er ihrer Auffassung entsprechend gegen die Werbebestimmungen des Heilmittelgesetzes verstossen hat, indem er ausführliche Beschreibungen von Yasmin, Evra, Nuva Ring, Mirena usw. auf seiner Homepage aufgeführt hat. Es handle sich in diesem Sinne nicht um Patientinneninformation sondern um Werbung und sei deshalb unzulässig. Es ist deshalb wichtig, dass jeder Einzelne seine Homepage bezüglich solcher Details kontrolliert.

\section{Aufklärungsprotokolle}

Die Aufklärungsprotokolle der SGGG wurden sorgfältig und teilweise in mühsamer Kleinarbeit zusammen mit der FMH und der Patientenorganisation ausgearbeitet. Die steigende Zahl der zivilrechtlichen Klagen in der Schweiz und das oft anspruchsvolle und fordernde Verhalten der Patientinnen, welche durch die Medien und Juristen gegen die Ärzte aufgestachelt werden, zeigen die Notwendigkeit der sorgfältigen schriftlichen präoperativen Aufklärung. Das gegenseitige Vertrauen als Basis einer guten Arzt-Patienten-Beziehung genügt in diesen Fällen nicht mehr. In den meisten öffentlichen Spitälern gehört die schriftliche Aufklärung der Patientin zur Regel; in den privaten Praxen besteht aber noch Nachholbedarf.

Diese Aufklärungsprotokolle, welche in deutscher, französischer und italienischer Sprache existieren, sind unter www. sggg.ch (Protokolle) abrufbar.

\section{Swissmom}

Durch die Empfehlung in der Praxis, im Spital, bei Hebammen und Mütterberaterinnen, aber auch durch Mundpropaganda und Medienberichte ist www.swissmom.ch zum grössten Informationsportal für alles rund ums Kinderkriegen und Kinderhaben geworden. Fast jede Schweizer Schwangere oder Mutter von Kleinkindern kennt Swissmom. Eine qualitativ vergleichbare Webseite, die von ärztlicher Seite unterstützt wird und einen so grossen Teil der Frauen erreicht, existiert in keinem anderen Land der Welt!

Die monatlichen Besuche nehmen stets zu. Waren es im Oktober 2006 noch 630000 (visitor sessions) sind es im Januar (per 14. Januar 2007) bereits 680000 . Über ein ganzes Jahr verteilt sind es durchschnittlich 24000 Besuche/Tag. Von den über 2500 Seiten wird der Schwangerschaftskalender «Woche für Woche» mit den jeweils relevanten Wochenthemen und Einblicken in die Entwicklung des Ungeborenen am meisten frequentiert. Das interaktive Forum verzeichnet pro Tag etwa 3000 neue Einträge, in Spitzenzeiten sind bis 270 Besucher gleichzeitig im Forum!

Dass ein so aktives Projekt etwas kostet, ist klar. Die SGGG hat Swissmom mit CHF 10 000.- unterstützt. Damit und mit der Bannerwerbung verschiedener Firmen können Webmaster und Server bezahlt werden. Aber weitere Sponsoren sind gesucht, und wir vom Vorstand der SGGG verdanken die Beträge bereits im Voraus herzlich.

\section{Jahreskongress 2007 in Lugano}

Der diesjährige Kongress steht unter dem Motto «Kongress für die praktische Ärztin, für den praktischen Arzt». Die Themenauswahl wurde den einzelnen Arbeitsgruppen überlassen.

Am ersten Tag wird die Schweizerische Gesellschaft für Reproduktionsmedizin zusammen mit der Arbeitsgemeinschaft für Endokrinologie und Reproduktionsmedizin das Thema polyzystisches Ovarialsyndrom/Infertilität behandeln. In diesen 
Vorträgen werden neue, für die Praxis relevante Erkenntnisse diskutiert. Am folgenden Morgen treffen sich die Urogynäkologen mit den Mitgliedern der endoskopischen Arbeitsgemeinschaft um über neue Methoden der Beckenbodenrekonstruktion zu diskutieren. Am Nachmittag werden uns zuerst die Mitglieder der Arbeitsgruppe für Fetomaternale Medizin über die aktuelle Überwachung des Feten vor und während der Geburt informieren und anschliessend die Mitglieder der Arbeitsgruppe für Onkologie über praktische Aspekte der Nachbehandlung bei Brustkrebs.

Die beiden Arbeitsgruppen Kolposkopie/Zervixpathologie und Kinder-/Jugendgynäkologie werden mit uns am Samstagmorgen die offiziellen Empfehlungen betreffend HPV-Impfung diskutieren. Abschliessen werden wir den Kongress mit dem
Thema der Sexualberatung in der gynäkologischen Sprechstunde, welches uns von den Mitgliedern der Arbeitsgemeinschaft für Psychosomatische Gynäkologie und Geburtshilfe vorgestellt wird.

Mit meinem Beitrag wollte ich Ihnen eine Übersicht über die aktuellen Probleme, Diskussionen und Projekte unserer Fachgesellschaft vermitteln. Ich bin überzeugt, dass wir durch proaktives Vorwärtsschauen und durch strategisches Denken vieles in positive Bahnen für uns und unsere Patientinnen bringen können.

In diesem Sinne wünsche ich ihnen allen ein erfolgreiches und glückliches 2007.

Prof. David Stucki Präsident SGGG 